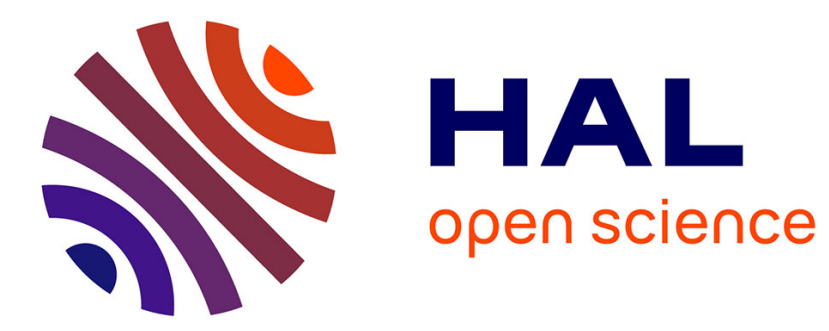

\title{
The random waypoint mobility model with uniform node spatial distribution
}

\author{
Dieter Mitsche, Giovanni Resta, Paolo Santi
}

\section{To cite this version:}

Dieter Mitsche, Giovanni Resta, Paolo Santi. The random waypoint mobility model with uniform node spatial distribution. Wireless Networks, 2013, pp.Print-ISSN : 1022-0038, Online-ISSN : $1572-$ 8196. 10.1007/s11276-013-0661-2 . hal-00923122

\section{HAL Id: hal-00923122 \\ https://hal.science/hal-00923122}

Submitted on 2 Jan 2014

HAL is a multi-disciplinary open access archive for the deposit and dissemination of scientific research documents, whether they are published or not. The documents may come from teaching and research institutions in France or abroad, or from public or private research centers.
L'archive ouverte pluridisciplinaire HAL, est destinée au dépôt et à la diffusion de documents scientifiques de niveau recherche, publiés ou non, émanant des établissements d'enseignement et de recherche français ou étrangers, des laboratoires publics ou privés. 


\title{
The Random Waypoint Mobility Model with Uniform Node Spatial Distribution
}

\author{
Dieter Mitsche · Giovanni Resta • Paolo \\ Santi
}

\begin{abstract}
In this paper, we tackle the problem of designing a random mobility model generating a target node spatial distribution. More specifically, we solve a long standing open problem by presenting two versions of the well-known random waypoint mobility model in bounded regions generating a uniform steady-state node spatial distribution. In the first version, named temporal- $R W P$, we exploit the temporal dimension of node mobility and achieve uniformity by continuously changing the speed of a mobile node as a function of its location and of the density function of trajectories in the movement region $R$. In the second version, named spatial- $R W P$, we instead exploit the spatial dimension and achieve uniformity by selecting waypoints according to a suitably defined mix of probability density functions. Both proposed models can be easily incorporated in wireless network simulators, and are thus of practical use.

The RWP models presented in this paper allow for the first time completely removing the well-known border effect causing possible inaccuracies in mobile network simulation, thus completing the picture of a "perfect" simulation methodology drawn in existing literature.
\end{abstract}

Keywords: Mobility modeling, random waypoint model, node spatial distribution, perfect simulation.

Dieter Mitsche

Ryerson University

350 Victoria Street, M5B2K3 Toronto - Canada

E-mail: dmitsche@ryerson.ca

Giovanni Resta

IIT - CNR

Via G. Moruzzi 1, 56124 Pisa - ITALY

E-mail: giovanni.resta@iit.cnr.it

Paolo Santi

IIT - CNR

Via G. Moruzzi 1, 56124 Pisa - ITALY

Tel.: +39-050-3152411

Fax: +39-050-3152333

E-mail: paolo.santi@iit.cnr.it 


\section{Introduction}

Mobility modeling has been a very active research field in the last decade. In fact, mobility is a fundamental component of the wireless network simulation process in most cases, and a deep understanding of the fundamental properties underlying node mobility is a pre-requisite for obtaining accurate simulation results. Furthermore, a characterization of the mobility model properties is the starting point for derivation of analytical results concerning mobile networks.

A striking example of the impact of mobility modeling on the wireless network simulation and analysis process is the random waypoint (RWP) mobility model [9], which still today is a commonly used mobility model in ad hoc network performance evaluation. A number of issues related to performance evaluation with RWP mobility have been reported in the literature. In particular, the node spatial distribution resulting from RWP mobility has been studied first through simulation [2-4] and then formally $[1,8]$, and occurrence of the so-called border effecti.e., of the fact that the concentration of RWP mobile nodes in a bounded region $R$ is sparser on the border than in the center of $R$ - has been reported. Another undesired effect of the RWP mobility model is the so-called speed decay phenomenon reported in [19], which refers to the fact that the average nodal speed measured at the beginning of the simulation is in general different (and higher) than that measured in stationary conditions.

The border effect and speed decay phenomenon impact simulation accuracy for a number of reasons. First, in both cases the initial conditions are different from those reached in steady state: the node spatial distribution is initially uniform (this is the standard way of selecting waypoints in the RWP model), while it is concentrated in the center of $R$ in stationary conditions; the average nodal speed is $v_{0}$ at the beginning of the simulation, while it is $v^{\prime} \leq v_{0}$ in stationary conditions, where $v^{\prime}=v_{0}$ only when the speed of a trip is chosen deterministically. Thus, in order to obtain accurate performance evaluation, gathering of simulation results should be started only after a relatively long period of (simulated) time, which results in considerable wastage of computational resources - see [19] for examples. The border effect has also significant influence on important networklevel parameters of a mobile network, such as the critical transmission range for connectivity (CTR) - i.e., the minimum common value of the transmission range the wireless nodes should use in order to keep the network connected [7]. In fact, it has been shown [18] that the CTR for connectivity in a RWP mobile network can be arbitrarily larger than that of a wireless network with uniform node spatial distribution. Thus, if, say, a routing protocol is evaluated in presence of RWP mobility and the transmission range is set as if nodes were uniformly distributed - which, again, is a quite common practice -, poor performance of the routing protocol is likely to be due to the fact that the underlying network topology becomes disconnected when nodes start moving, instead of being caused by some shortcoming in the routing protocol itself.

For the reasons described above, significant efforts have been devoted in the literature to carefully accounting for the border effect and speed decay phenomena, and to identifying a "perfect" simulation methodology. For instance, in [20] the authors define a methodology for initializing the simulation directly in stationary conditions, thus removing the speed decay phenomenon. More recently, a similar "perfect" simulation methodology encompassing not only the average nodal speed 
distribution but also node spatial distribution has been defined in [11] for a wider class of mobility models, including RWP.

While the "perfect" simulation methodology allows initializing a mobile network directly in its stationary conditions, the problem of how to design random mobility models such that certain desired stationary conditions are achieved has remained mostly unaddressed so far. This problem is very relevant, since solving it might allow the definition of mobility models that couple the simplicity and simulation efficiency of random mobility models with the "realism" of recently proposed trace-based mobility models (e.g., $[12,15,16]$ ). If one is able to arbitrarily shape the stationary properties of a simple random mobility model, the model can be made to fit properties observed in real-world traces, while minimally impacting its simplicity and ease-of-use.

As an instance of this problem, we show in this paper how to modify the RWP mobility model so that the generated node spatial distribution is different from the one generated by the standard model. More specifically, the targeted node spatial distribution is the uniform distribution, which has been considered in the literature as a desirable property for a mobility model [6].

In this paper, we show how the node spatial distribution of the RWP mobility model can be arbitrarily shaped (subject to a rotary symmetric condition) by exploiting two orthogonal approaches, aimed at modifying the temporal or spatial dimension of the mobility model. The first approach is based on continuously varying node velocity based on its position, while in the second approach the distribution of waypoints is suitably defined so to give rise to a uniform spatial distribution of mobile nodes. As we shall see, our "uniform" versions of the RWP mobility model only minimally impact the simplicity of the model, and are then very easy to implement in a wireless simulator.

While the temporal and spatial approaches presented in this paper have been specifically tailored to a specific target node spatial distribution (uniform) and mobility model (RWP), these approaches can be readily applied to define RWP extensions with arbitrary rotary-symmetric node spatial distributions. Most importantly, the tool and techniques presented in this paper can be the starting point for achieving arbitrary node spatial distribution in other random mobility models such as Random Direction, Random Walk, etc.

Thus, the wealth of theoretical tools and methodologies presented in this paper can be considered as complementing the "perfect simulation" methodology described in [11] with the ability of arbitrarily shaping the node spatial distribution generated by a mobility model. We remark here that node spatial distribution is a very important feature of a mobility model, since fundamental network parameters such as network topology, network connectivity, etc., depends on it.

\section{Related work and contributions}

The first property of RWP mobility that has been studied in the literature is the node spatial distribution, which led to the discovery of the border effect when nodes move in a bounded region. The node spatial distribution of RWP mobile networks has been first studied through simulations - see, e.g., [2-4] -, and then formally. In particular, in [1] the authors presented the exact, closed form expression of the RWP node spatial distribution in one-dimensional networks, and a 
close approximation of the distribution resulting when $R$ is the unit square. Later on, the authors of [8] extended the results in [1] presenting the exact RWP node spatial distribution for arbitrary two-dimensional, bounded, convex shapes of $R$, and arbitrary waypoint distributions.

The speed decay phenomenon has been first reported in [19], and more thoroughly analyzed in [20], where the authors presented also a "perfect" simulation methodology to initialize a RWP mobile network directly in stationary conditions - for what concerns the average nodal speed -, thus avoiding the possibly very long transient regime. Later on, the authors of [11] have introduced a theoretical framework for analyzing a large class of mobility models including RWP, and stated conditions under which the models can be proved to admit a stationary regime. Furthermore, the authors of [11] extended the "perfect" simulation methodology to this larger class of mobility models, and to encompass not only average nodal speed, but also node spatial distribution.

Despite the extensive work reported above, the specific problem considered in this paper is remained open so far: Is it possible to define a version of RWP mobility in a bounded region generating a uniform node spatial distribution? This problem has been explicitly mentioned as open in [8], where the authors hint to existence of such a RWP model based on the different shapes of the node spatial distribution resulting when waypoints are uniformly distributed in the unit disk, or on the border of the unit disk. This observation is the starting point of our work on the spatial version of uniform RWP presented in Section 5.

The work that is more similar to ours is [6], where the authors present a framework for studying mobility properties based on partial differential equations. The framework presented in [6] allows not only characterizing mobility properties such as node spatial distribution once mobility parameters are fixed, but also to "reverse engineer" the setting of mobility parameters resulting in a certain desired node spatial distribution. However, the authors of [6] apply such "reverse engineering" approach only to the random direction model, while they leave open - due to the involved analytical difficulties - the problem of "reverse engineering" the RWP model.

In this paper, we at least partially address the above open problem by "reverse engineering" the RWP model in order to obtain a specific node spatial distribution, namely the uniform node spatial distribution. While the methodologies presented in this paper are specifically devised to obtain a uniform node spatial distribution, the spatial approach presented in Section 5 is amenable to generalization to other target (rotary symmetric) node spatial distributions on the unit disk. More in general, some of the ideas presented in this paper to achieve a uniform RWP mobility model such as location-dependent definition of speed or pause time, can prove useful in the analysis and design of random mobility models in general.

\section{Preliminaries}

The well-known RWP model, first introduced in [9], is defined as follows: Given a convex region $R$, a point (waypoint) $P_{1}$ is selected uniformly at random in $R$, and a pause time at $P_{1}$ is chosen uniformly at random in an interval $\left[t_{\text {min }}, t_{\text {max }}\right]$; upon termination of pause time, a new waypoint $P_{2}$ is chosen uniformly at random in $R$, and the node starts moving from $P_{1}$ to $P_{2}$ along a straight line trajectory with 
a speed chosen uniformly at random in an interval $\left[v_{\min }, v_{\max }\right]$. When the node arrives at destination, the pause and movement process is repeated as above.

The above rules define movement of a single node. Since movements of different nodes are independent in the RWP model, a mobile network with $n$ nodes is modeled through $n$ identical and independent stochastic mobility processes corresponding to the above described movement pattern. More formally, the stochastic process underlying RWP mobility is defined as follows (see, e.g., $[1,8]$ ):

$$
\left\{D_{i}, T_{i}, V_{i}\right\}
$$

where $i \in \mathbb{N}$ is an index parameter corresponding to the $i$-th trip, $D_{i}$ is a random variable corresponding to the destination of the $i$-th trip, $T_{i}$ is a random variable corresponding to the pause time at $D_{i}$, and $V_{i}$ is a random variable corresponding to the velocity of the node in the $i$-th trip. Note that an additional random variable $D_{0}$ corresponding to the location of the initial waypoint is needed in order to fully describe the stochastic process underlying RWP mobility.

The stationary node spatial distribution generated by RWP mobility is formally defined as follows ${ }^{1}$ : let $X_{t}=\left(x_{t}, y_{t}\right)$ be the random variable corresponding to the position of the mobile node at time $t$, and let $f_{X(t)}(x, y)$ be the probability density function (pdf) of $X_{t}$; the stationary node spatial distribution of the RWP mobility model is defined as the pdf

$$
f_{X}(x, y)=\lim _{t \rightarrow \infty} f_{X(t)}(x, y) .
$$

Informally speaking, the stationary node spatial distribution can be interpreted as the pdf describing the position of a mobile node at a random (and sufficiently large) instant of time $t$. The formal existence of a stationary node spatial distribution for the RWP model (i.e., the existence of the above limit) has been first established in [17] under some assumptions, and then proved in a more general mobility framework in [11].

The node spatial distribution of the original RWP model and of some of its variants has been extensively studied in the literature - see $[1,8]$. However, current approaches fix the distribution of waypoints and of the other mobility parameters first, and then evaluate the resulting node spatial distribution. In this paper, we take a radically different approach: we first fix the desired node spatial distribution, and then investigate how to modify some parameters of the RWP model in order to obtain the desired spatial distribution. In particular, our goal in the following is defining versions of the RWP mobility model generating uniform (or a close approximation of uniform) node spatial distribution; i.e., our goal is having $f_{X}(x, y)=U(R)$, where $U(R)$ is the uniform distribution on $R$, defined as $U(R)=1 / A(R)$, where $A(R)$ is the area of $R$.

To achieve our goal, we will present two approaches, one leveraging the temporal and the other the spatial dimension of the mobility model. In the first approach, the speed of a node is continuously varied during its trip to the destination depending on its position in $R$. This approach has the advantage of producing a provably uniform node spatial distribution with arbitrary convex shapes of $R$ and arbitrary pause time distributions; however, the continuously varying speed part of the model is not in accordance with most types of real-world mobility. The

\footnotetext{
1 For ease of notation, we are assuming $R$ is a two-dimensional region.
} 


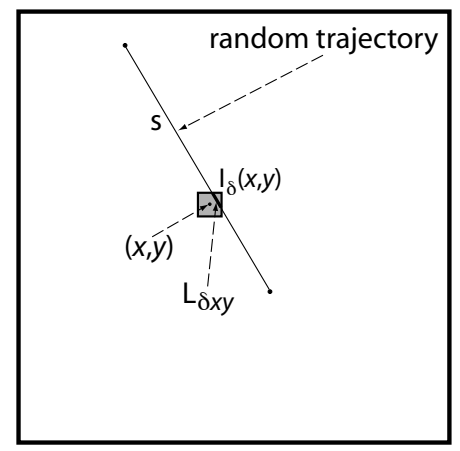

Fig. 1 Deriving the node spatial distribution of the standard RWP model.

second approach solves this issue by retaining the standard RWP assumption of fixed velocity during a trip, and by exploiting the spatial dimension instead - i.e., modifying the way waypoints are selected. In particular, waypoints are selected through a suitably weighted mix of probability distributions taken from a family $\mathcal{F}$ of pdfs. While characterizing properties of the node spatial densities corresponding to waypoint densities in $\mathcal{F}$ and derivation of the corresponding weights is highly nontrivial, the resulting method for generating RWP mobility with uniform node spatial distribution is very easy to implement and of practical use for wireless network simulation.

Before ending this section, we want to comment about impact of the proposed RWP variants on the realism of the mobility model. Historically, the RWP model represented a breakthrough with respect to existing models such as random walks since it introduced the notion of (straight line) trajectory in the mobility model. Due to its simplicity and improved realism, RWP has become the most commonly used model in ad hoc wireless network simulation. The two versions of RWP proposed herein preserve the notion of trajectory, while changing the way speed is chosen during a trip (temporal-RWP), or the way waypoints are selected (spatialRWP). While we acknowledge that the assumption of continuously changing speed done in the former model might affect its realism, the latter model preserves intact the same level of realism of the original RWP model. In fact, the assumption of uniformly distributed waypoints is an artifact of the original model motivated by simplicity, and it is not supported by observations of human mobility. On the contrary, analysis of mobility traces has recently shown that selection of waypoints is not uniform in space [10]. While we do not claim here that the waypoint selection method presented in this paper is more adherent to reality than that of the original RWP model, we claim instead that our proposed waypoint selection method is at least as realistic as the one of the original model based on waypoint uniformity.

\section{The Temporal-RWP model}

The first variation of the original RWP model we propose is motivated by the following simple, yet striking, observation. Due to the mean ergodicity property of 
the stochastic movement process [1], the probability density $f_{X}(x, y)$ is determined as the limit, for $\delta \rightarrow 0$, of the ratio $\frac{P_{\delta}(x, y)}{g(\delta)}$, where $P_{\delta}(x, y)$ is the probability of finding the mobile node in a small region $I_{\delta}(x, y)$ centered at $(x, y)$ at an arbitrary (but large enough) time instant $t$, and $g(\delta)$ is the area of $I_{\delta}(x, y)$ - say, a square of side $\delta$ centered at $(x, y)^{2}$. In turn, probability $P_{\delta}(x, y)$ can be computed as the expectation of the length $L_{\delta, x, y}$ of the intersection between a random segment in $R$ and $I_{\delta}(x, y)$, divided by the expected length $E[L]$ of a random segment in $R$. Referring to Figure 1, the segment $S$ represents a segment with endpoints chosen uniformly at random in $R$, while the part of the segment in bold represents the intersection of $S$ with region $I_{\delta}(x, y)$. Since in the standard RWP mobility model, although different speeds can be selected in different trips, the speed is kept fixed in each single trip, the amount of space covered by a node in a unit of time is constant during a trip. Thus, under this assumption, the spatial and temporal domains become equivalent, and the amount of time a mobile node spends in $I_{\delta}(x, y)$ on its way to the destination - which is the quantity to be evaluated to determine the node spatial distribution - becomes equivalent to the amount of space (length) within region $I_{\delta}(x, y)$ covered by the trajectory.

This equivalence between the temporal and spatial domain is the very reason why the border effect occurs in the original RWP model. Let $f_{S}(x, y)$ denote the density of trajectories resulting from the standard RWP model with uniformly selected waypoint - see $[1,8]$ for closed-form approximations and exact implicit characterization of $f_{S}(x, y)$ for different shapes of $R$. Under the assumption of uniformly distributed waypoints and bounded region $R$, the density of trajectories crossing $I_{\delta}(x, y)$ - spatial dimension - is not uniform and is concentrated in the center of the $R$, and since spatial and temporal dimension are equivalent in the original RWP model, it follows that also the node spatial distribution - determined by the temporal dimension - is not uniform. Thus, in order to eliminate the border effect completely, it is sufficient to remove this equivalence between the temporal and spatial dimension, and to introduce instead a direct correlation between the temporal and the spatial dimension of mobility.

Our goal is obtaining a uniform density of the node spatial distribution which, despite the misleading name, is indeed determined by the amount of time a node spends in $I_{\delta}(x, y)$. In particular, we have:

$$
f_{X}(x, y)=\lim _{\delta \rightarrow 0} \frac{P_{\delta}(x, y)}{g(\delta)}=\lim _{\delta \rightarrow 0} \frac{\frac{E\left[T_{\delta, x, y}\right]}{E[T]}}{g(\delta)}
$$

where $E[T]$ is the expected duration of a trip, and $E\left[T_{\delta, x, y}\right]$ is the expected time a node spends in the region $I_{\delta}(x, y)$. Since once all parameters of the mobility model are set the value of $E[T]$ is a constant that does not depend on $(x, y)$, and the same holds for quantity $g(\delta)$, the only term in the above expression depending on spatial coordinates $(x, y)$ is $E\left[T_{\delta, x, y}\right]$. So, if RWP mobility rules can be defined so that $E\left[T_{\delta, x, y}\right]$ does not depend on $(x, y)$, we have that the resulting node spatial distribution $f_{X}(x, y)$ becomes independent of $(x, y)$ as well, i.e., uniform up to normalization.

\footnotetext{
2 Formally speaking, region $I_{\delta}(x, y)$ should be defined as the intersection of a square with region $R$, to account for the effect of border. However, in the following the effect of border which does not impact the reasoning underlying the definition of the temporal-RWP model is disregarded to keep notation and presentation simpler.
} 
The time a node spends in $I_{\delta}(x, y)$ depends on the length of the trajectory intersecting $I_{\delta}(x, y)$ and on the node velocity, formally:

$$
T_{\delta, x, y}=\frac{L_{\delta, x, y}}{V_{\delta, x, y}}
$$

where in the formula above we have made explicit the fact that the velocity $V_{\delta, x, y}$ of a node while traveling through $I_{\delta}(x, y)$ might depend on $(x, y)$. Since the trajectory density $f_{S}$ is given by

$$
f_{S}(x, y)=\lim _{\delta \rightarrow 0} \frac{\frac{E\left[L_{\delta, x, y}\right]}{E[L]}}{g(\delta)},
$$

and the expected length $E[L]$ of the trajectory is also a constant once fixed the mobility parameters, we can write (up to constant values):

$$
f_{X}(x, y)=\lim _{\delta \rightarrow 0} \frac{\frac{E\left[\frac{\int_{I_{\delta}(x, y)} f_{S}(u, v) d u d v}{V_{\delta, x, y}}\right]}{E[T]}}{g(\delta)} .
$$

In order to make $f_{X}(x, y)$ uniform, it is then sufficient to set

$$
V_{\delta, x, y}=v_{\delta, x, y}=k \cdot \int_{I_{\delta}(x, y)} f_{S}(u, v) d u d v
$$

and

$$
V_{x, y}=v_{x, y}=\lim _{\delta \rightarrow 0} V_{\delta, x, y}=k \cdot f_{S}(x, y)
$$

where $k$ is a constant that can be used to set the desired speed range (see below), and we have used lower case notation for the speed to indicate that nodal speed is not a random variable, but it is deterministically defined given the location of the node in $R$.

Thus, in the above described model, speed is changed in a continuous way during a node's trip to destination, as a function of its position in $R$. In particular, speed is directly proportional to the trajectory density $f_{S}$, resulting in relatively higher speeds in the center of the region, and in a speed approaching zero when the node approaches the border. Thus, even if a node travels more frequently in the center of $R$, it has a correspondingly higher speed when in the center of $R$, so that the resulting time the node spends in each subregion of $R$ is constant.

We can then state the following theorem:

Theorem 1 Let $R$ be an arbitrary bounded, open, convex region, and assume a mobile node moves in $R$ according to the RWP mobility model with uniformly distributed waypoints and arbitrary pause time distribution. Let $f_{S}(x, y)$ be the pdf describing the spatial density of trajectories in $R$, and assume the speed of a node in a trip is continuously changed based on its location according to the following rule: $v_{x, y}=k \cdot f_{S}(x, y)$, for some $k>0$, where $v_{x, y}$ represents the velocity of the node when located at coordinates $(x, y) \in R$. Then, the resulting steady state node spatial distribution is uniform. 


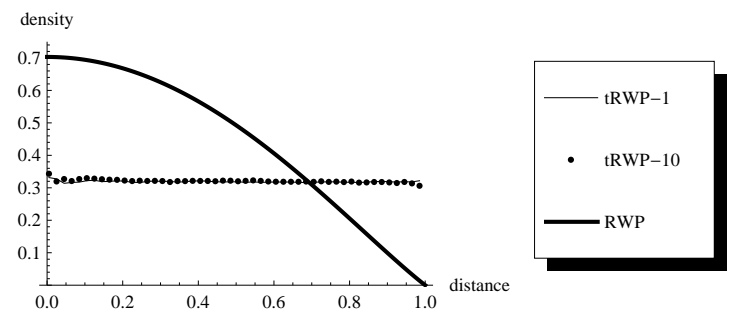

Fig. 2 The node spatial distribution $f_{R W P}$ of the original RWP model (RWP) and of the discretized temporal RWP model with time step of 1 sec (tRWP-1) and 10 sec (tRWP-10).

Proof The proof follows from the derivations above, and from the observation that, under the hypotheses of the theorem, the position of a node while pausing at a waypoint is uniformly distributed. Thus, both the mobility and the pause component of the node spatial distribution are uniformly distributed, and the resultant node spatial distribution is uniform independently of the weights (determined by the specific pause time distribution) of the two components.

Notice that the temporal-RWP model belongs to the class of random trip models as defined in [11] (formal proof omitted due to lack of space), so convergence of the node spatial distribution to the stationary regime is guaranteed.

\subsection{The Temporal-RWP model in simulations}

We now shortly describe how the temporal-RWP model can be implemented in a simulator, and show that the generated node spatial distribution is uniform. For ease of presentation, we assume $R$ to be the unit disk.

The density of trajectories (which is equivalent to the node spatial distribution in the standard RWP model) when waypoints are uniformly distributed in the unit disk has been derived in [8], and has the shape reported in Figure 2. Due to rotational symmetry of the distribution, the pdf is reported in Figure 2 in polar coordinates, and is a function $f_{R W P}(r, \theta)=f_{R W P}(r)$ of the distance of a point from the origin.

The temporal RWP model is implemented as follows: first, the desired range of speeds is selected. We observe that the maximum of $f_{R W P}(r)$ is 0.7031 and is achieved at $r=0$, while the minimum of $f_{R W P}(r)$ is 0 and is achieved at $r=1$. Thus, the minimum speed in the temporal RWP model is always 0 , and is achieved when a node hits the border (note that this happens with vanishing probability). The maximum speed can instead be arbitrarily chosen by properly setting $k$. For instance, if a maximum speed of $3 \mathrm{~m} / \mathrm{sec}$ is desired (pedestrian mobility), it is sufficient to set $k=3 / 0.7031=4.267$.

In order to implement the temporal RWP mobility, it is sufficient to use a sufficiently small time step in the simulation: at each time step, we first check whether the mobile node is in pause or movement state; if the node is in movement state and its current position is $\left(x_{t}, y_{t}\right)$, the speed for the next time step is set to $v_{x_{t}, y_{t}}$. The node spatial distribution of the temporal RWP model when the diameter of the disk is set to $1 \mathrm{Km}$, the maximum speed to $2 \mathrm{~m} / \mathrm{sec}$ (pedestrian mobility), and 
different time steps are used is reported in Figure 2. As seen from the figure, even with a relatively large time step of $10 \mathrm{sec}$ the node spatial distribution generated by the temporal-RWP model is indistinguishable from uniform distribution, thus showing that the time and speed discretization introduced in the simulation has virtually no effect on the node spatial distribution of the temporal-RWP model.

\section{The Spatial-RWP Model}

The temporal-RWP model introduced in the previous section generates a provably uniform node spatial distribution under quite general conditions. However, the continuously changing speed assumption is scarcely representative of real-world mobility. To solve this problem, in this section we present a version of RWP mobility achieving near-uniform node spatial distribution under the standard assumption of fixed (although possibly randomly chosen) speed during a trip.

We recall that, under the fixed speed assumption, the temporal and spatial dimension of mobility are equivalent, and the pdf of density of trajectories in $R$ is equivalent to the node spatial distribution $f_{X}$. For this reason, in the following, as customary in the literature on RWP mobility, we use the term node spatial distribution to refer to the density of trajectories as well. Furthermore, in what follows we assume $R$ is the unit disk, and pause time is set to 0 (i.e., only the mobility component of the distribution is present). The assumption of 0 pause time will be released at the end of this section.

The starting point of our spatial approach to uniform RWP mobility is the observation made in [8] that the complementary shapes of node spatial distributions of the standard RWP model and of a version of RWP in which waypoints are uniformly distributed on the border of the disk (see Figure 4) suggest that it should be possible to define a waypoint density function generating a uniform node spatial distribution.

The direct way of deriving such waypoint density function would be solving the following integral equation, which equals the node spatial distribution of a RWP model with arbitrary waypoint density $f_{w}(r)$ - see [8] - to the uniform distribution:

$$
\begin{aligned}
\frac{1}{\pi}= & \frac{1}{E[\ell]} \int_{0}^{2 \pi} d \theta \int_{0}^{a_{2}(r, \theta)} d r_{2} \\
& \int_{0}^{a_{1}(r, \theta)} d r_{1}\left(r_{1}+r_{2}\right) \cdot f_{w}\left(r_{1}\right) \cdot f_{w}\left(r_{2}\right),
\end{aligned}
$$

where $f_{w}(r)$ is the unknown, rotary symmetric waypoint density function, $E[\ell]$ is the expected length of a random segment with endpoints chosen according to $f_{w}(r)$, and $a_{1}(r, \theta), a_{2}(r, \theta)$ are defined as follows:

$$
\begin{aligned}
& a_{1}(r, \theta)=\sqrt{1-r^{2} \cos ^{2} \theta}-r \sin \theta \\
& a_{2}(r, \theta)=\sqrt{1-r^{2} \cos ^{2} \theta}+r \sin \theta .
\end{aligned}
$$

Unfortunately, equation (1) is a complex integral equation in non-standard form, and cannot be solved with standard techniques. In the following, we present 


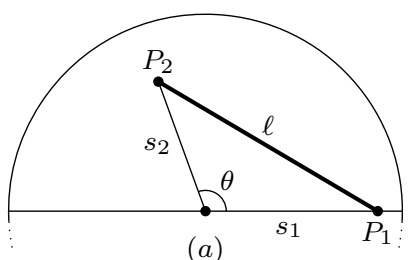

(a)

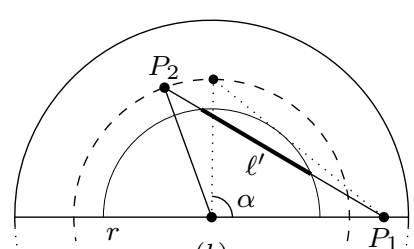

(b)

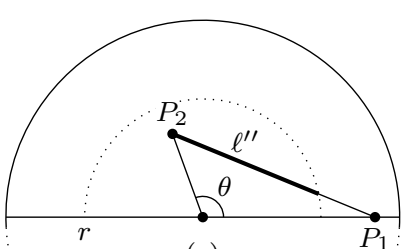

(c)

Fig. 3 Illustrations used in the derivation of $F_{X_{h}}$.

a method for closely approximating $f_{w}(r)$. The idea is to use a finite family $\mathcal{F}=\left\{f_{0}, f_{1}, \ldots\right\}$ of waypoint density functions resulting in diverse shapes of the resulting node spatial distributions $\mathcal{F}_{X}=\left\{f_{X_{0}}, f_{X_{1}}, \ldots\right\}$, and to compute a linear combination $\alpha_{0} f_{X_{0}}+\alpha_{1} f_{X_{1}}+\ldots$ of the functions in $\mathcal{F}_{X}$ that closely approximates the uniform node spatial distribution. Note that we compute the $\alpha_{i}$ s not from the initial family $\mathcal{F}$, but rather from the resulting node spatial distributions $\mathcal{F}_{X}$. Thus, we can exploit the linearity of the integral operator. Up to some technical tricks in waypoint selection, this implies that the corresponding linear combination $\alpha_{0} f_{0}+\alpha_{1} f_{1}+\ldots$ of waypoint density functions in $\mathcal{F}$ generates a near-uniform node spatial distribution.

5.1 Deriving the family of node spatial distributions

Family $\mathcal{F}_{h}$ is defined as follows:

$$
\mathcal{F}_{h}=\left\{f_{0}, f_{1}, \ldots, f_{h}\right\}
$$

where $h \geq 1$ is an integer parameter, and $f_{i}$ is the uniform distribution over the region of points at distance at least $i / h$ from the origin, i.e.

$$
f_{i}(r)=\left\{\begin{array}{ll}
\frac{1}{\pi\left(1-\left(\frac{i}{h}\right)^{2}\right)} & \text { if } r \geq \frac{i}{h} \\
0 & \text { otherwise }
\end{array},\right.
$$

for $0 \leq i<h$, and

$$
f_{h}(r)=\left\{\begin{array}{ll}
\frac{1}{2 \pi} & \text { if } r=1 \\
0 & \text { otherwise }
\end{array} .\right.
$$

It is easy to see that, for any $h, f_{0}$ corresponds to the uniform distribution on $R$ and $f_{h}$ corresponds to the uniform distribution on the border of the disk.

The node spatial distributions $f_{X_{0}}, f_{X_{h}}$ corresponding to $f_{0}$ and $f_{h}$ have been derived in [8]. In what follows, we present the derivation of $f_{X_{i}}$ for a generic value of $i$. Indeed, the derivation is for a generic waypoint density $f_{z}$ where waypoints are chosen uniformly at distance at least $z$ from the origin, where $0<z<1$ is an arbitrary real number.

The derivation is based on the standard approach of conditioning on specific positions of the waypoints, and then computing the integral of the resulting conditional density for all possible positions of the waypoints. Indeed, following the derivation of $f_{X_{h}}$ in [8], we proceed to derive the cumulative density $F_{X_{z}}(r)$ - i.e., the probability of finding a mobile node at distance $\leq r$ from the origin - , from which density $f_{X_{z}}$ can be easily obtained through derivation. 
Let us consider waypoints $P_{1}=\left(s_{1}, 0\right)$ and $P_{2}=\left(s_{2}, \theta\right)$, chosen uniformly at random at distances $\geq z$ from the origin, and assume w.l.o.g. that $z \leq s_{1}, s_{2} \leq 1$, as depicted in Figure 3.a. In the following, we use $t=s_{1}^{2}+s_{2}^{2}-2 s_{1} s_{2} \cos \theta$ as an abbreviation. We first compute the average length $L_{z}$ of the random segment joining $P_{1}$ and $P_{2}$, which is given by

$$
L_{z}=\int_{z}^{1} \int_{z}^{1} \int_{0}^{2 \pi} \ell\left(s_{1}, s_{2}, \theta\right) w\left(s_{1}, s_{2}, z\right) \mathrm{d} \theta \mathrm{d} s_{2} \mathrm{~d} s_{1},
$$

where $\ell\left(s_{1}, s_{2}, \theta\right)=\sqrt{t}$ is the distance between $P_{1}$ and $P_{2}$ and

$$
w\left(s_{1}, s_{2}, z\right)=\frac{1}{2 \pi} \frac{2 \pi s_{1}}{\pi\left(1-z^{2}\right)} \frac{2 \pi s_{2}}{\pi\left(1-z^{2}\right)}=\frac{2 s_{1} s_{2}}{\pi\left(1-z^{2}\right)^{2}}
$$

is the product of the probability densities for $\theta, s_{1}$ and $s_{2}$.

In order to compute the average length $D_{z}(r)$ of the intersection of the segment $\overline{P_{1} P_{2}}$ with a disk of radius $r$, we should distinguish 4 cases.

1) $r \leq z \leq s_{1}, s_{2} \leq 1$. Here

$$
D_{1, z}(r)=2 \int_{z}^{1} \int_{z}^{1} \int_{\alpha}^{\pi} \bar{\ell}\left(P_{1}, P_{2}, r\right) w\left(s_{1}, s_{2}, z\right) \mathrm{d} \theta \mathrm{d} s_{2} \mathrm{~d} s_{1},
$$

where $\bar{\ell}\left(P_{1}, P_{2}, r\right)=2 \sqrt{r^{2}-\left(s_{1} s_{2} \sin \theta\right) / t}$ and

$$
\alpha=\arccos \left(\frac{r^{2}-\sqrt{\left(s_{1}^{2}-r^{2}\right)\left(s_{2}^{2}-r^{2}\right)}}{s_{1} s_{2}}\right),
$$

is the angle such that, for $\alpha \leq \theta \leq 2 \pi-\alpha$, there exists a non-empty intersection between the segment $\overline{P_{1} P_{2}}$ and the disk of radius $r$ (see Fig.3.b).

2) $z \leq r \leq s_{1}, s_{2} \leq 1$. Similarly to the previous case, we have

$$
D_{2, z}(r)=2 \int_{r}^{1} \int_{r}^{1} \int_{\alpha}^{\pi} \bar{\ell}\left(P_{1}, P_{2}, r\right) w\left(s_{1}, s_{2}, z\right) \mathrm{d} \theta \mathrm{d} s_{2} \mathrm{~d} s_{1},
$$

3) $z \leq s_{2} \leq r \leq s_{1} \leq 1$ and the symmetric case with $s_{1} \leq s_{2}$. As depicted in Fig.3.c, we have

$$
D_{3, z}(r)=4 \int_{r}^{1} \int_{z}^{r} \int_{0}^{\pi} \hat{\ell}\left(P_{1}, P_{2}, r\right) w\left(s_{1}, s_{2}, z\right) \mathrm{d} \theta \mathrm{d} s_{2} \mathrm{~d} s_{1},
$$

where

$$
\hat{\ell}\left(P_{1}, P_{2}, r\right)=\sqrt{r^{2}-s_{1}^{2}+\frac{t+u}{2}-\frac{\left(s_{1}^{2}-s_{2}^{2}\right)\left(u-s_{1}^{2}+s_{2}^{2}\right)}{2 t},}
$$

setting $u=2 \sqrt{r^{2} t-s_{1}^{2} s_{2}^{2} \sin ^{2}(\theta)}$ for brevity.

4) $z \leq s_{1}, s_{2} \leq r \leq 1$. The whole segment $\overline{P_{1} P_{2}}$ lies in the disk of radius $r$, thus we can apply the same formula used for $L_{z}$ and obtain

$$
D_{4, z}(r)=\int_{z}^{r} \int_{z}^{r} \int_{0}^{2 \pi} \ell\left(s_{1}, s_{2}, \theta\right) w\left(s_{1}, s_{2}, z\right) d \theta d s_{2} d s_{1} .
$$




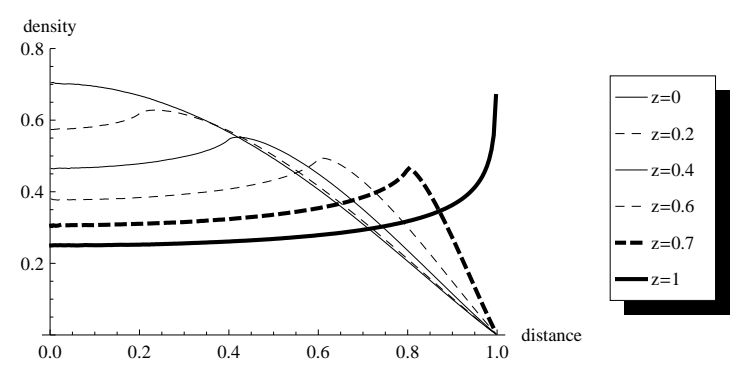

Fig. 4 Node spatial distribution $f_{X_{z}}(r)$ of the RWP model with waypoint distribution $f_{z}(r)$, for different values of $z$.

Summarizing, the cumulative density function $F_{X_{z}}(r)$ is defined as

$$
F_{X_{z}}(r)=\left\{\begin{array}{ll}
\frac{D_{1, z}(r)}{L_{z}} & \text { if } 0 \leq r \leq z \\
\frac{D_{2, z}(r)+D_{3, z}(r)+D_{4, z}(r)}{L_{z}} & \text { if } z<r \leq 1
\end{array},\right.
$$

and the node spatial density $f_{X_{z}}(r)$ can be easily obtained from $F_{X_{z}}(r)$ by derivation with respect to $r$. The shape of $f_{X_{z}}(r)$ for different values of $z$ is reported in Figure 4. Note that when $z=0$ we have the standard RWP model, while $z=1$ corresponds to RWP with waypoints uniformly chosen on the border.

\subsection{Computing the mix of waypoint distributions}

Let us consider a family $\mathcal{F}_{h}=\left\{f_{0}, \ldots, f_{h}\right\}$ of waypoint density functions, and the corresponding family $\mathcal{F}_{X}=\left\{f_{X_{0}}, \ldots, f_{X_{h}}\right\}$ of node spatial distributions. Our goal in the following is determining a set of non-negative weights $\alpha_{0}, \alpha_{1}, \ldots$ such that function $f_{X, \alpha}=\sum_{i} \alpha_{i} f_{X_{i}}$ is as close to uniform as possible. Let $\bar{\alpha}_{0}, \bar{\alpha}_{1}, \ldots$ be the set of such "uniforming" weights - we will see in the following how to determine this set -, and assume that waypoints are selected according to the following mix of probability densities: $f_{\alpha}=\sum_{i} \bar{\alpha}_{i} f_{i}$. Up to some technical trick in the selection of waypoints as described below, due to the linearity of the integral operator the node spatial distribution resulting when waypoints are chosen according to $f_{\alpha}$ is exactly $f_{X, \alpha}$, i.e., very close to uniform.

Two observations are in order before proceeding further. First, distributing waypoints according to a mix of probability density functions is an easy task, which amounts to: $i$ ) normalizing weights $\bar{\alpha}_{i}$ to achieve $\sum_{i} \bar{\alpha}_{i}=1 ; i i$ ) extracting uniformly at random a real number $w$ in the [0,1] interval; iii) finding the index $j$ such that $\sum_{i<j} \bar{\alpha}_{i}<w \leq \sum_{i<j+1} \bar{\alpha}_{i}$; and $\left.i v\right)$ selecting the next waypoint according to $f_{j}$.

The second observation concerns the waypoints selection method. In fact, the straightforward approach of choosing the new waypoint at each step according to probability density $f_{\alpha}$ indeed generates a node spatial distribution quite different from $f_{X, \alpha}$, for the following reason. Let $P_{1}, P_{2}, P_{3}, \ldots$ be the series of waypoints selected by a mobile node. If $f_{\alpha}$ is used at each step to select the next waypoint, the event "waypoint $P_{\ell}$ is chosen according to density $f_{j}$, and waypoint $P_{\ell+1}$ is chosen according to density $f_{j^{\prime}}$, with $j^{\prime} \neq j$ ", which we call a mix event, becomes very 
likely. Occurrence of mix events causes inaccuracies in estimating the node spatial distribution generated by $f_{\alpha}$ with $f_{X, \alpha}$, due to the fact that the single node spatial distributions $f_{X_{i}}$ used as components of $f_{X, \alpha}$ are computed under the assumption that both the starting and the ending point of a trajectory are chosen according to the same waypoint density $f_{i}$. In other words, the $f_{X_{i}}$ s are computed conditioning on occurrence of the complementary of a mix event. To deterministically reduce occurrence of mix events, it is sufficient to choose waypoints in batches: once a certain waypoint density $f_{j}$ is selected out of the density mix according to the above described procedure, a batch of $s$ consecutive waypoints are chosen according to $f_{j}$. This simple trick is sufficient to ensure that mix events occur at most once every $s$ consecutive waypoint selections. Thus, the node spatial distribution generated when $s$ consecutive waypoints are selected according to $f_{\alpha}$ becomes closer to $f_{X, \alpha}$ as $s$ increases.

The value of $s$ to be used in simulations should be carefully evaluated: on one hand, using a large value of $s$ is desirable for generating a node spatial distribution closely resembling $f_{X, \alpha}$, but it has the disadvantage of inducing a very long transient regime before stationary conditions are reached; on the other hand, a small value of $s$ is desirable for shortening the duration of the transient regime and simulation running time, but it has the negative effect of generating a node spatial distribution quite different from the desired distribution $f_{X, \alpha}$. In the following, we will show that using a small value of $s=4$ is already sufficient to obtain a very close approximation of $f_{X, \alpha}$ and, hence, of the uniform node spatial distribution.

In order to closely approximate the uniform node spatial distribution, we have considered a quite large family of waypoint distributions, namely $\mathcal{F}_{100}$. However, in order to reduce the complexity of the "optimal" weight searching process, we have imposed the condition that only $u$ of the $\alpha_{i}$ weights can be non-zero, where $u$ is a tunable parameter. In other words, our goal in the following is: $i$ ) selecting a set of $u$ spatial densities $\bar{f}_{X_{1}}, \ldots, \bar{f}_{X_{u}}$ in the family $\mathcal{F}_{X}$ of spatial densities corresponding to waypoint densities in $\mathcal{F}_{100}$; and $i i$ ) determine a set of positive weights $\bar{\alpha}_{i}$ for densities $\bar{f}_{X_{1}}, \ldots, \bar{f}_{X_{u}}$ such that the corresponding node spatial density $f_{X, \alpha}=\sum_{i=1}^{u} \bar{\alpha}_{i} \cdot \bar{f}_{X_{i}}$ is as close to uniform as possible.

The iterative local search algorithm used to select the set of density and corresponding weights is reported in Figure 5. Initially, a candidate set of spatial density functions $\mathcal{F}^{\prime}$ is randomly selected from $\mathcal{F}_{X}$, and the set of weights that minimizes the Mean Square Error (MSE) with respect to uniform given $\mathcal{F}^{\prime}$ is computed using the least square method on a set of 200 equally spaced points. Then, a local search is done with the purpose of finding a pair of density functions $f_{h}, f_{k}$ which can substitute two densities in $\mathcal{F}^{\prime}$ and improve the MSE (steps 6-11). This process is repeated until no further MSE improvement can be achieved. The node spatial distribution obtained at the end of the iterative local search algorithm for different values of $u$ is reported in Figure 6, and the corresponding set of candidate functions with relative weights are reported in Table 1 . As seen from the figure, a candidate set of 6 densities is already sufficient to obtain a spatial distribution $F_{X, \alpha}$ closely resembling uniform distribution.

It is worth observing that an exhaustive search of all possible combinations of $u$ out of 101 elements in $\mathcal{F}_{X}$ and computation of the optimal set of weights is computationally feasible only for very small values of $u$ - up to 6 . For such small values of $u$, we have verified that the iterative local search algorithm of Figure 5 always finds the optimal solution. For larger values of $u$, the iterative local search 


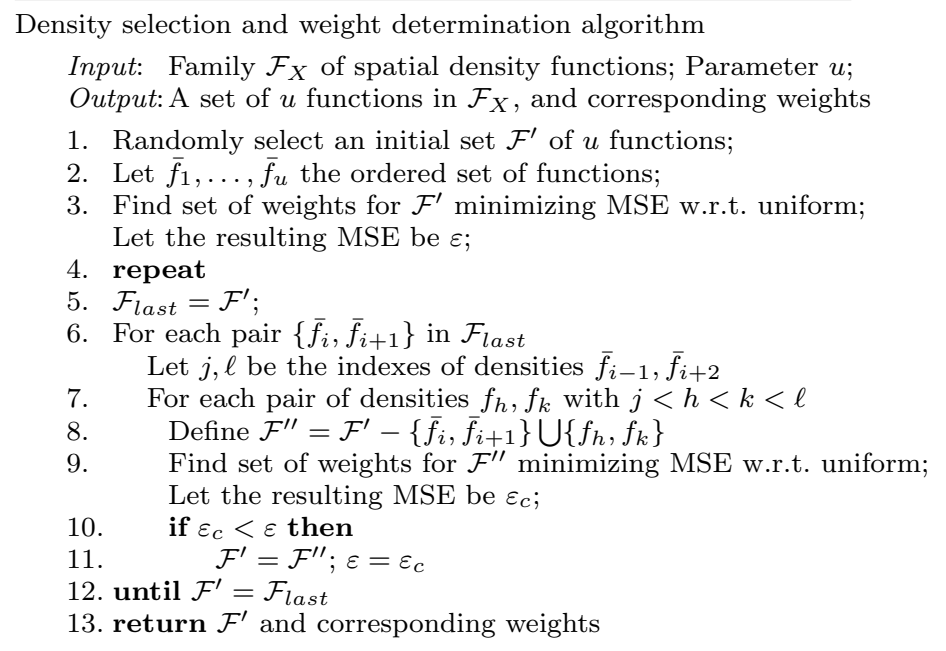

Fig. 5 The spatial density selection and weight determination algorithm.

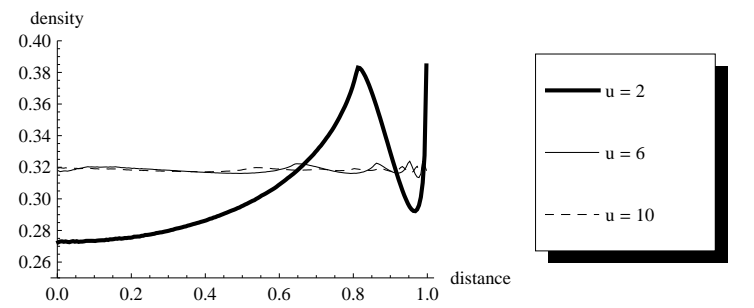

Fig. 6 Node spatial distribution $f_{X, \alpha}(r)$ obtained with the "optimal" mix of weights for different number $u$ of distributions in the candidate set.

\begin{tabular}{|c|c|c|}
\hline$u$ & Density functions & weights \\
\hline 2 & $f_{X_{81}}, f_{X_{100}}$ & $0.43,0.57$ \\
6 & $f_{X_{5}}, f_{X_{64}}, f_{X_{86}}$, & $0.12,0.10,0.10$, \\
& $f_{X_{95}}, f_{X_{99}}, f_{X_{100}}$ & $0.11,0.13,0.44$ \\
10 & $f_{X_{0}}, f_{X_{52}}, f_{X_{66}}, f_{X_{70}}$, & $0.11,0.06,0.004,0.04$ \\
& $f_{X_{80}}, f_{X_{87}}, f_{X_{93}}$, & $0.047,0.053,0.07$, \\
& $f_{X_{97}}, f_{X_{99}}, f_{X_{100}}$ & $0.072,0.104,0.44$ \\
\hline
\end{tabular}

Table 1 Density functions and weights corresponding to the spatial densities reported in Figure 6.

algorithm of Figure 5 cannot be directly compared with the optimal solution. This is the reason why we use the term "optimal" to refer to the set of density functions and corresponding weights returned by our iterative local search algorithm. 


\subsection{Stationarity of the Spatial-RWP model}

Our goal is to show that the Spatial-RWP model introduced above has a stationary distribution to which the distribution converges after sufficiently many steps (in the sense of total variation distance, that is, in the sense of the largest possible difference between the probabilities that two probability measures can assign to the same event). Since the points on the unit disk represent an uncountable state space, we first have to establish the necessary theory for generalized Markov chains in order to state the theorem under which a stationary distribution occurs.

Let $(S, \mathcal{S})$ be a measurable space, that is, $S$ is a state space, and $\mathcal{S}$ be a $\sigma$-algebra defined on it (that is, a non-empty set of subsets of $S$ closed under complement and countable unions). Let $p: \mathcal{S} \times \mathcal{S} \rightarrow \mathbb{R}$ be a transition probability, that is, for each $x \in S, A \rightarrow p(x, A)$ is a probability measure on $(S, \mathcal{S})$, and for each $A \in \mathcal{S}, x \rightarrow p(x, A)$ is a measurable function. Recall that, given a measurable space, a filtration is an increasing sequence of $\sigma$-algebras $\left\{\mathcal{F}_{t}\right\}_{t \geq 0}$, where $\mathcal{F}_{t} \subseteq$ $\mathcal{S}$ for any $t$ and $t_{1} \leq t_{2}$ implies $\mathcal{F}_{t_{1}} \subseteq \mathcal{F}_{t_{2}}$. Informally speaking, $\mathcal{F}_{t}$ represents the information up to time $t$, and this information is increasing. A sequence of random variables $X_{n}$ is a Markov chain with respect to a given filtration $\mathcal{F}_{n}=$ $\sigma\left(X_{0}, \ldots, X_{n}\right)$ with transition probability $p$ if $\mathcal{P}\left(X_{n+1} \in B \mid \mathcal{F}_{n}\right)=p\left(X_{n}, B\right)$ for any $B \in \mathcal{S}$. Denote also by $\mathcal{P}_{x}$ the probability measure short for $\mathcal{P}_{\delta_{x}}$, that is, the measure concentrated on the point $x$.

The following definition can be found in [5]:

Definition 1 A Markov chain $X_{n}$ is a Harris chain if one can find $A, B \in \mathcal{S}$, a function $q$ with $q(x, y) \geq \epsilon>0$ for $x \in A, y \in B$, and a probability measure $\rho$ concentrated on $B$ such that

- If $\tau_{A}=\inf \left\{n \geq 0: X_{n} \in A\right\}$, then $\mathcal{P}_{z}\left(\tau_{A}<\infty\right)>0$ for all $z \in S$.

- If $x \in A$ and $C \subset B$, then $p(x, C) \geq \int_{C} q(x, y) \rho(d y)$.

Theorem 2 Spatial-RWP has a stationary distribution $\pi$, and starting from any distribution, the distribution converges to $\pi$ (convergence in the sense of total variation distance).

Proof Let $X_{n}$ be the random variable accounting for the position of the $n$-th waypoint (the sequence $X_{n}$ is space-continuous, but time-discrete). The idea of the proof is the following: our goal is to first verify the conditions of being a Harris chain, and then we will use a well-known theorem stating that, under certain conditions, this Harris chain converges to its stationary distribution.

In our particular case, $S$ represents the open unit disk on $\mathbb{R}^{2}$, and we can take $\mathcal{S}$ to be the Borel sets on this disk, that is, the $\sigma$-algebra generated by all products of closed (say) intervals in this disk. At any step, knowing the previous final waypoint and the distribution this waypoint was chosen from (more precisely, in our filtration we also have to know the batch size $s$, and we also have to know the index $j \leq s$, corresponding to the fact that it was the $j$-th waypoint chosen from the batch of the current distribution), the distribution of the next waypoint does not depend on previous waypoints. Thus, if $\mathcal{F}_{n}$ is the $\sigma$-algebra generated by this information, the sequence of random variables $\left\{X_{n}\right\}_{n \geq 1}$ is a Markov chain. Moreover, for any pair of distributions the start waypoint and the end waypoint are chosen from and for any batch index $j \leq s$, there is always a positive probability density $p$ (depending on the distributions, and the batch index) to go from any 
particular point $x$ to any other particular point $y$. In particular, since for all choices of distributions the corresponding spatial waypoint distributions are differentiable, they are continuous. Thus, there is a transition probability satisfying $p(x, d y)=$ $p(x, y) d y$ where $(x, y) \rightarrow p(x, y)$ is continuous. Since this holds for any $x$ and $y$, we can choose $A$ and $B$ in Definition 1 to be the whole unit disk. We know that $p(x, y) \geq \epsilon>0$ on $A \times B$, and by letting $\rho(C)=|B \cap C| /|B|$, where $|B|$ is the Lebesgue measure on $B$, the conditions above hold, and hence $X_{n}$ is a Harris chain. For our particular case of $A$ being the whole unit disk, starting from the Harris chain we can construct a Markov chain $\bar{X}_{n}$ by slightly modifying the transition probabilities: for each $x \in A$, with probability $\epsilon>0$ go to a new state $\alpha$ (that is, $\bar{p}(x,\{\alpha\})=\epsilon$ ), and for each other state $C \in \mathcal{S}$, set the new probability $\bar{p}(x, C)=p(x, C)-\epsilon \rho(C)$ ( $\rho$ again being the Lebesgue measure on $C$ ), and for $x=\alpha, \bar{p}(\alpha, D)=\int \rho(d x) \bar{p}(x, D)$ for any state $D \in\{B, B \cup\{\alpha\}, B \in \mathcal{S}\}$. Note in particular that this implies $\bar{p}(\alpha, \alpha)=\epsilon$.

Let now $R=\inf \left\{n \geq 1: \bar{X}_{n}=\alpha\right\}$. A chain is called recurrent if $\mathcal{P}_{\alpha}(R<$ $\infty)=1$. In our case, the probability to go to state $\alpha$ is $\epsilon$ in each step (independent of the current state), and thus our Harris chain is clearly recurrent. By Theorem 6.8.5 of [5], there is a stationary measure $\pi$ on our Harris chain. Moreover, a Harris chain is aperiodic, if the greatest common divisor of the set $\left\{n \geq 1: \bar{p}^{n}(\alpha, \alpha)>0\right\}$ is 1 . Given the fact that we have $\bar{p}(\alpha, \alpha)>0$, our Harris chain is aperiodic. Finally, denote by $\|\cdot\|$ the total variation distance between two probability measures. Since in our case $\mathcal{P}_{x}(R<\infty)=1$ for any $x$, by Theorem 6.8 .8 of [5], as $n \rightarrow \infty$,

$$
\left\|\bar{p}^{n}(x, \cdot)-\pi(\cdot)\right\| \rightarrow 0,
$$

which ends the proof of the theorem.

\subsection{The Spatial-RWP model in simulations}

We now summarize how the Spatial-RWP model can be used in simulations, and evaluate the effect of waypoint batch size on the node spatial distribution. In the Spatial-RWP model, pause time is set to 0, and the speed of a trip is chosen uniformly at random in an interval $\left[v_{\min }, v_{\max }\right]$. The first batch of $s$ waypoints is chosen according to a weighted mix of waypoint density functions, as described is Section 5.2. The density functions used in the mix are the waypoint densities corresponding to the spatial densities reported in Table 1. For instance, if 6 densities are used to form the mix, chosen waypoint densities are $f_{5}, f_{64}, f_{86}, f_{95}, f_{99}$ and $f_{100}$. These functions are weighted according to the normalized weights reported in Table 1. Once the last waypoint in the batch is reached, a new density function for selecting the next $s$ waypoints is selected according to the described mix, and the $s$ waypoints are chosen. This process is then repeated over and over. The node spatial distribution resulting from the Spatial-RWP model with 6 densities and different batch sizes $s$ is reported in Figure 7. The node spatial distribution has been estimated by running a large number of experiments (more than $10^{6}$ ), where each experiment consisted in performing a number of mobility steps according to the spatial-RWP model sufficient to make at least 50 trips on the average, and in recording the position of the node at the end of the experiment. The figure reports also the node spatial distribution with Spatial-RWP mobility with 2 densities and batch size of 8 and, for comparison, the distribution with the original RWP model. 


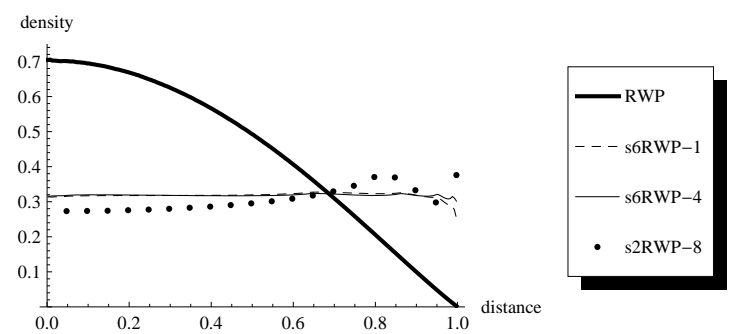

Fig. 7 Node spatial distribution of the Spatial-RWP model with 6 densities and batch size 1 (s6RWP-1) and 4 (s6RWP-4), and with 2 densities and batch size 8 (s2RWP-8). For comparison, the node spatial distribution of the standard RWP model is also reported (RWP).

As seen from the figure, the effect of batch size on the resulting node spatial distribution is perceivable, especially close to the border: with a batch size of 1 and 6 densities, the node spatial distribution is almost uniform, except for a perceivable drop close to the border; with a larger batch size of 4 , this drop is considerably smoothed, and the node spatial distribution becomes very close to uniform. Much more noticeable is the effect of the number of waypoint densities used to select waypoints: if only 2 densities are used to select waypoints - see Table 1 for the specific densities and weights used -, even with batch size of 8 we obtain a node spatial distribution which is considerably different from uniform. Thus, our results suggest that using 6 waypoint densities with batch size of 4 is a suitable choice for generating a node spatial distribution very close to uniform.

To further validate this claim, fixed the number $n$ of nodes in the network, we have computed the critical transmission range (CTR) for connectivity when nodes are uniformly distributed, move according to the standard RWP model with 0 pause time, or move according to the Spatial-RWP model with 6 densities and batch size 4 . The CTR for connectivity is estimated by letting the mobile networks move for a time $T$ necessary to complete about 50 trips on the average, and then computing the longest edge of the Minimum Euclidean Spanning Tree built on the node positions after time $T$. In case of uniformly distributed nodes, the longest MST edge is computed immediately. These random experiments are repeated a large number of times $\left(10^{5}\right)$, and the CTR for connectivity is finally estimated as the $99 \%$ quantile of the empirically generated distribution of the longest MST edge. The results of our experiments when nodes are deployed in the unit disk are reported in Table 2. As seen from the table, the CTR for connectivity with standard RWP mobility is smaller than with uniform distribution when $n=25$, and larger than the uniform CTR when $n=100$ and $n=500$. This is due to the fact that, as observed in [18], when $n$ is small there is a very low probability of finding at least one node close to the border, hence node concentration in the center of the disk favors lower CTR values; as $n$ increases, though, the probability of finding at least one node close to the border increases as well, leading to CTR values higher than with uniform node distribution. In accordance with [18], the relative difference between the CTR with uniform node distribution and with RWP mobility increases with $n$ : it is $7.6 \%$ when $n=100$, and it becomes $37.8 \%$ when $n=$ 500. On the contrary, the CTR with s-RWP mobility is almost indistinguishable from that with uniform node distribution independently of the value of $n$. 


\begin{tabular}{|c|c|c|c|}
\hline Model & CTR, $n=25$ & CTR, $n=100$ & CTR, $n=500$ \\
\hline Unif & 0.7397 & 0.3857 & 0.1789 \\
RWP & 0.6592 & 0.4152 & 0.2466 \\
s-RWP & 0.7378 & 0.3856 & 0.1798 \\
\hline
\end{tabular}

Table 2 Values of the CTR for connectivity with uniformly distributed nodes, and with RWP and Spatial-RWP mobile networks.

\subsection{The Spatial-RWP with non-zero pause times}

We now explain how to define a mix of waypoint density functions such that the resulting spatial node distribution becomes very close to the uniform distribution in the general case of non-zero pause time at the waypoints.

In the case of non-zero pause time, the node spatial distribution is composed of two components [1]: the mobility component, which accounts for the fraction of time the node is moving, and the pause component, which accounts for the fraction of time the node is resting at waypoints. These components are weighted by non-negative weights $w_{1}, w_{2}$ with $w_{1}+w_{2}=1$, representing the expected fraction of time the node is moving or resting at a waypoint. Earlier in this paper, we have shown how to make the mobility component uniform, namely, by making the waypoint distribution non-uniform. Thus, directly using the above defined waypoint distribution, when combined with a uniform non-zero pause time distribution, yields a non-uniform node spatial node distribution, due to the fact that some waypoints are chosen with higher probability density than others, and, consequently, the total pause times at these waypoints are longer than those at less frequently selected waypoints.

At least two options are at hand in order to solve this problem. First, it is possible to modify the pause time distribution making it a function of the distance $d$ from the waypoint to the center of $R$. Choose the waypoint distribution as in the case of zero pause time, and let $f_{X}(d)$ be the (non-uniform) resulting density of choosing a waypoint of distance $0 \leq d \leq 1$ from the origin. Choose now the pause time $p(d)$ at a waypoint at distance $0 \leq d \leq 1$ from the origin such that the expected time of pausing at the waypoint (which can be computed as the product of the waypoint density $f_{X}(d)$ and the pause time $\left.p(d)\right)$ is constant over $d$. In the simplest possible case, $p(d)$ is deterministically chosen as $p(d)=c / f_{X}(d)$, for some universal constant $c>0$. In words, at points which are visited more often, the pause time is shorter, so that the expected duration of the pause time at any waypoint in $R$ remains constant. This way, both the mobility and the pause component of the node spatial distribution become uniform, and the resulting node spatial distribution turns out to be uniformly independently of the values of $w_{1}$ and $w_{2}$.

If distance-dependent pause time distribution cannot be used, then the mobility component can still be shaped in such a way that the resulting spatial node distribution is uniform: first, using the notation of Section V, given an arbitrary but fixed waypoint density $f_{i}$, exactly as in the case with zero pause time, one computes the resulting spatial node density corresponding to $f_{X_{i}}$. To this density one now has to add the pause time to all waypoints which can be chosen in this distribution, that is, $\bar{f}_{X_{i}}(d)=f_{X_{i}}(d)$, if $d<\frac{i}{h}$, and $\bar{f}_{X_{i}}(d)=f_{X_{i}}(d)+p$, for 
$\frac{i}{h} \leq d \leq 1$, where $p$ is the pause time at any waypoint. We then normalize these values in order to obtain a new distribution $\hat{f}_{X_{i}}$, and for these new distributions one then computes suitable weights $\alpha_{i}$ such that $f=\sum_{i=0}^{h} \alpha_{i} \hat{f}_{X_{i}}$ is as close to uniform as possible. Using the same methodology as before, we finally use these $\alpha_{i}$ as probabilities to choose $f_{i}$.

\section{The Perfect Simulation}

In the previous sections, we have seen how to modify the RWP model in order to remove the border effect. On the other hand, speed decay caused by RWP mobility can be removed as described in [20]. An interesting question to investigate is whether there exists a methodology that allows to simultaneously remove both these effects, enabling so called "perfect simulation", i.e., a simulation methodology where the mobile system is initialized directly in the stationary regime and node spatial distribution is uniform ${ }^{3}$.

In what follows, we describe how to devise a "perfect simulation" methodology using the spatial-RWP model presented in Section 5. A similar methodology can be defined for the temporal-RWP model, which is not reported due to lack of space.

In the following, to simplify presentation, we present a version of the "perfect simulation" methodology introduced in [11] customized to the spatial-RWP model with zero pause time. The "perfect simulation" methodology is summarized below:

1. Determine the initial trajectory: first, fixed a value of $s$ (batch size) in the spatial-RWP model, determine whether the two initial waypoints belong to different batches (with probability $\frac{1}{s}$ ), or to the same batch (with probability $\left.1-\frac{1}{s}\right)$.

1.1. If the two waypoints belong to different batches, select waypoints $W_{0}$ and $W_{1}$ according to the mix of waypoint distributions defined in Section 5; set initialBatchSize to $s$;

1.2. If the two waypoints belong to the same batch, then select one of the waypoint density functions $f_{j}$ in the mix based on the $\alpha$ weights, and select both waypoints $W_{0}$ and $W_{1}$ according to $f_{j}$. Select an integer $i$ uniformly at random in $\{1, \ldots, s-1\}$, and set initialBatchSize to $i$;

2 Determine the initial speed: select a random speed $v$ using density function

$$
f_{V}(v)=\frac{1}{v \ln \left(\frac{v_{\max }}{v_{\min }}\right)},
$$

as defined in [20].

3. System initialization: select a number $u$ uniformly at random in the $[0,1]$ interval; initialize the node speed to $v$, the node next waypoint to $W_{1}$, and the node current position along the segment connecting $W_{0}$ with $W_{1}$, at distance

\footnotetext{
3 Notice that the notion of "perfect simulation" considered in [11] accounts only for the existence of a stationary regime, and for the initialization of the mobile network directly into this regime. Indeed, for the reasons explained in the Introduction, we are interested herein in a stronger notion of "perfect simulation" where, besides the above properties, the additional property that the stationary node spatial distribution is uniform must be fulfilled.
} 
$u \cdot \operatorname{dist}\left(W_{0}, W_{1}\right)$ from $W_{0}$, where $\operatorname{dist}(x, y)$ is the Euclidean distance between $x$ and $y$.

4. Movement phase: after initialization, rules for selecting waypoints and speed are as in the spatial-RWP model, with the only difference that the first batch of selected waypoints has size $i$ instead of $s$.

Based on the results presented in $[11,20]$, and on our characterization of stationary properties of the node spatial distribution of the spatial-RWP model, we can conclude that the above described methodology initializes the mobile system directly into the stationary regime, and that the stationary regime of the node spatial distribution is the target distribution, namely, the uniform distribution on the disk. Thus, no "warming up" period is needed when performing simulations, and important network parameters such as the node transmission range need not to be changed with respect to the case of uniform node spatial distribution.

\section{Concluding Remarks}

In this paper, we have addressed the problem of designing a random mobility model generating a desired node spatial distribution. More specifically, we have addressed the long standing open problem of designing a variant of the well-known RWP mobility model generating a uniform node spatial distribution. We have proposed two approaches to achieve this goal, named temporal- and spatial-RWP model. While the temporal-RWP model can be used when mobile nodes are deployed in regions of arbitrary convex shapes, and generates a uniform node spatial distribution with arbitrary pause time distributions at waypoints, the spatial-RWP model is shown to produce uniform node spatial distribution under the more stringent assumption that the movement region is a disk.

The approaches proposed in this paper to achieve a target node spatial distribution can be extended to deal with different target distributions and generalize to other random mobility models. For instance, the methodology used to derive the spatial-RWP model can be immediately extended to define versions of RWP mobility generating arbitrary, rotary symmetric distributions: to this purpose, it is sufficient to use a different target spatial distribution in the iterative local search algorithm reported in Figure 5. If the target node spatial distribution is not rotary symmetric, a rotational asymmetric family of candidate waypoint distributions should be used instead of family $\mathcal{F}_{100}$. Thus, the ideas and techniques presented in this paper can prove useful also to define versions of RWP mobility resulting in any target, non-uniform node spatial distribution, as those observed in real-world traces (see, e.g., [10]).

\section{References}

1. C. Bettstetter, G. Resta, P. Santi, "The Node Distribution of the Random Waypoint Mobility Model for Wireless Ad Hoc Networks", IEEE Trans. on Mobile Computing, Vol. 2, n.3, pp. 257-269, July-Sept. 2003.

2. C. Bettstetter, H. Hartenstein, X. Perez-Costa, "Stochastic Properties of the Random Waypoint Mobility Mdoel", Wireless Networks, Vol. 10, n. 5, pp. 555-567, 2004.

3. C. Bettstetter, O. Krause, "On Border Effects in Modeling and Simulation of Wireless Ad Hoc Networks", Proc. IEEE Conf. on Mobile and Wireless Communication and Networks (MWCN), pp. 20-27, 2001. 
4. D.M.Blough, G.Resta, P.Santi, "A Statistical Analysis of the Long-Run Node Spatial Distribution in Mobile Ad Hoc Networks", Wireless Networks, Vol. 10, pp. 543-554, 2004.

5. R. Durrett, Probability: Theory and Examples, 4th Edition, Cambridge University Press, June 2010

6. M. Garetto, E. Leonardi, "Analysis of Random Mobility Models with PDEs", Proc. ACM MobiHoc, pp. 73-84, 2006.

7. P. Gupta, P.R. Kumar, "Critical Power for Asymptotic Connectivity in Wireless Networks", Stochastic Analysis, Control, Optimization and Applications, Birkhauser, Boston, pp. 547-566, 1998.

8. E. Hyytiä, P. Lassila, J. Virtamo, "Spatial Node Distribution of the Random Waypoint Mobility Model with Applications", IEEE Trans. on Mobile Computing, Vol. 5, n. 6, pp. 680-694, 2006.

9. D. B. Johnson, D. A. Maltz, "Dynamic source routing in ad hoc wireless networks", in Mobile Computing, Kluwer Academic Publishers, pp. 153-181, 1996.

10. M. Kim, D. Kotz D, S. Kim, "Extracting a mobility model from real user traces", Proc. IEEE Infocom, pp. 1-13, 2006.

11. J.-Y. LeBoudec, M. Vojnovic, "The Random Trip Model: Stability, Stationary Regime, and Perfect Simulation", ACM/IEEE Trans. on Networking, Vol. 14, n. 6, pp. 1153-1166, 2006.

12. K. Lee, S. Hong, S. Kim, I. Rhee, S. Chong, "SLAW: A New Mobility Model for Human Walks", Proc. IEEE Infocom, pp. 855-863, 2009.

13. Z. Li, H. Shen, "A Hierarchical Account-Aided Reputation Management System for LargeScale MANETs", Proc. IEEE Infocom, pp. 909-917, 2011.

14. V. Manfredi, M. Crovella, J. Kurose, "Understanding Stateful vs. Stateless Communication Strategies for Ad Hoc Networks", Proc. ACM Mobicom, pp. 313-324, 2011.

15. A. Mei, J. Stefa, "SWIM: A Simple Model to Generate Small Mobile Worlds", Proc. IEEE Infocom, pp. 2106-2113, 2009.

16. M. Musolesi, C. Mascolo, "Designing Mobility Models Based on Social Network Theory", Mobile Computing and Communications Review, Vol. 11, pp. 1-11, 2007.

17. W. Navidi, T. Camp, "Stationary Distributions for the Random Waypoint Mobility Model", IEEE Trans. on Mobile Computing, Vol. 3, n. 1, pp. 99-108, 2004.

18. P. Santi, "The Critical Transmitting Range for Connectivity in Mobile Ad Hoc Networks", IEEE Transactions on Mobile Computing, Vol. 4, No. 3, pp. 310-317, 2005.

19. J. Yoon, M. Liu, B. Noble, "Random Waypoint Considered Harmful", Proc. IEEE Infocom, pp. 1312-1321, 2003.

20. J. Yoon, M. Liu, B. Noble, "Sound mobility models" Proc. ACM Mobicom, pp. 205-216, 2003. 